\title{
Uncovering the transcriptional signatures of hub connectivity in neural networks
}

\author{
Aurina Arnatkevičiūtè ${ }^{1 *}$, Ben D. Fulcher ${ }^{1,2}$, Alex Fornito ${ }^{1}$ \\ 1 Brain and Mental Health Research Hub, Monash Institute of Cognitive and Clinical Neurosciences, School of \\ Psychological Sciences, Monash University, 770 Blackburn Rd, Clayton, 3168, VIC, Australia. \\ 2 School of Physics, Sydney University, Sydney, 2006, NSW, Australia. \\ * aurina.arnatkeviciute@monash.edu
}

\begin{abstract}
Connections in nervous systems are disproportionately concentrated on a small subset of neural elements that act as network hubs. Hubs have been found across different of species and scales ranging from C.elegans to mouse, rat, cat, macaque and human, suggesting a role for genetic influences. The recent availability of brain-wide gene expression atlases provides new opportunities for mapping the transcriptional correlates of large-scale network-level phenotypes. Here we review studies that use these atlases to investigate gene expression patterns associated with hub connectivity in neural networks and present evidence that some of these patterns are conserved across species and scales.
\end{abstract}

\section{Keywords}

Connectome, hub, rich club, gene expression, network neuroscience, graph theory, genome.

\section{Introduction}

The brain is a multiscale network, with neuronal elements exhibiting coordinated patterns of activity that unfold across several orders of magnitude in time and space (Buzsáki and Draguhn, 2004, Lichtman and Denk, 2011, Fornito et al. 2016). Graph theory provides a useful approach to represent network organization at each scale by focusing on the essential elements of the system: processing units and their interactions, represented respectively as nodes and edges in the graph (Bullmore and Sporns, 2009, Fornito et al., 2016). The advantage of using a graph theoretic approach to understand the organizational properties of the brain is that the same analysis tools can be applied regardless of the species or scale, ranging from electron micrograph data of neuron-and-synapse connectivity in the nematode worm Caenorhabditis elegans (White et al., 1986, Varshney et al., 2011), through tract-tracing data in the mouse (Oh et al. 2014, Gămănuţ et al., 2018) and macaque (Stephan et al., 2001, Markov et al., 2014), to brain-wide non-invasive structural and functional imaging in the human (Bassett and Bullmore, 2009, Bullmore and Sporns, 2009, Fornito et al. 2013 ).

A growing body of work has demonstrated that the connection topology of neural networks - that is, the specific arrangement of connections between system elements-shows a number of non-random properties that are conserved across different scales and in different species (Bullmore and Sporns, 2009, Sporns, 2011, Fornito et al., 2016, van den Heuvel et al., 2016a, Schröter et al., 2017). These include (i) a predominance of short-range, locally clustered connections supporting functional specialization coupled with sparse, long-range projections that may promote global integration and functional diversity, resulting in an economical small-world organization (Watts and Strogatz, 1998, Bassett and Bullmore, 2017, Betzel and Bassett, 2017); (ii) the presence of densely connected sub-networks, termed modules, organized hierarchically across several resolution levels so that modules contain nested sub-modules and so on (Meunier et al. 2009, Bassett et al., 2010); (iii) a fat-tailed distribution of connectivity across nodes, such that some nodes possess a relatively large number of connections and act as network hubs (van den Heuvel and Sporns, 2011, Towlson et al., 2013, van den Heuvel et al., 2016a) and (iv) a dense inter-connectivity of hub nodes, leading to the 
formation of a 'rich club' (Zamora-López et al., 2010, van den Heuvel and Sporns, 2011, Harriger et al., 2012, Towlson et al. 2013).

The strong conservation of such topological properties across scales and species implies a role for genes in shaping network organization. Twin studies have shown that topological properties of human brain networks mapped at the macroscale are heritable (Smit et al., 2008, Fornito et al., 2011, van den Heuvel et al., 2013, Bohlken et al., 2014, Sinclair et al., 2015, Zhan et al., 2015, Colclough et al., 2017), but they do not indicate the specific genes involved. Studies linking structural variation in the genome to variability in network-level phenotypes, both at the level of candidate genes (Liu et al., 2010, Brown et al., 2011, Dennis et al., 2011, Markett et al., 2017) and in genome-wide scans (Jahanshad et al. 2013), have started to address this gap. However, they provide a partial picture, as it is often unclear how a given DNA variant impacts gene expression to give rise to phenotypic variability.

In neuroscience, it has been difficult to link direct measures of gene expression to variation in network phenotypes defined across large swathes of the brain, as gene expression has traditionally only been quantifiable though invasive interrogation of regionally localized tissue samples. The recent availability of large-scale, brain-wide atlases of gene expression (Lein et al. 2007. Hawrylycz et al., 2012), have overcome this hurdle and presented new opportunities to understand the molecular correlates of network-level phenotypes. Patterns of gene expression have been used to predict whether two neurons (or large-scale brain regions) will be structurally connected (Varadan et al., 2006, Kaufman et al., 2006, Baruch et al., 2008, Wolf et al., 2011, French and Pavlidis, 2011, Ji et al., 2014, Fakhry and Ji, 2015), and confirmed that regional variations in gene expression track specific aspects of structural (Goel et al., 2014. Forest) et al. 2017, Parkes et al., 2017, Romero-Garcia et al., 2018) and functional (Cioli et al., 2014, Richiardi et al. 2015, Hawrylycz et al., 2015, Krienen et al., 2016, Anderson et al., 2018) brain networks. The integration of gene expression atlases with imaging data is also shedding light on the molecular correlates of macroscopic brain changes observed in a range of disorders such as Huntington's (McColgan et al., 2018), Parkinson's (Rittman et al., 2016) and schizophrenia (Romme et al. 2017).

One important aspect of brain network organization relies is the distribution of connections across nodes, which is disproportionately concentrated on a small number of network hubs (van den Heuvel and Sporns, 2011, Towlson et al. 2013). Most simply, network hubs are defined as nodes with a relatively large number of connections, placing them in a topologically central position within the network [although other definitions are possible; see (Power et al., 2011. Oldham et al., 2018)]. Intuitively, the global air transportation network offers insight into the role of hubs in mediating network traffic flow; certain airports, such as Dubai International, London Heathrow, and LAX are linked to the rest of the network by a much larger number of direct flights than other airports. They are thus positioned to mediate a large fraction of intercontinental travel. Similarly, connections are not distributed equally across neurons, neuronal populations or large brain areas, with specific network elements possessing the lion's share of connections (van den Heuvel and Sporns, 2011, Towlson et al., 2013, de Reus and van den Heuvel, 2014, van den Heuvel et al. 2016a). These brain hubs are thought to play a critical role in the functional integration of anatomically disparate systems (Harriger et al. 2012. van den Heuvel et al. 2012), and are disproportionately impacted by a diverse variety of brain diseases (Crossley et al. 2014, Fornito et al. 2015). Thus, understanding the molecular basis for hub connectivity may provide insight not only into integrated cerebral function, but also into the various disease processes that plague the brain.

In this article, we review how brain-wide gene expression atlases have been used to link two traditionally disparate scales of analysis in neuroscience: molecular function (microscale) and whole-brain network topology (macroscale), by identifying the transcriptional correlates of brain network hubs. We begin with a brief overview of the expression atlases that are currently available then consider how hubs are defined in brain networks and what we know about their functional role. We then examine research indicating that brain network hubs possess a distinct and conserved transcriptional signature.

\section{Characterizing gene expression across the entire brain}

Gene expression is a process through which genetic information encoded in sequences of DNA is read and used to synthesize a particular gene product. The two key steps in this complex process are transcription, where an unwound segment of DNA is read to produce messenger (mRNA), and translation, which occurs when the resulting mRNA is used to synthesize the gene product, such as a protein. Gene expression is commonly inferred from mRNA levels, thus serving as an index of transcriptional activity - an indirect proxy for the protein abundance. Transcriptional activity can be measured using several different techniques that either assay bulk tissue samples [microarray (Schulze and 
Downward, 2001), RNA-seq (Mortazavi et al., 2008, Wang et al., 2009)], histological sections at a cellular resolution [in situ hybridisation (ISH) (Schulze and Downward, 2001)], or single cells [single-cell RNA sequencing (scRNA-seq) (Tang et al. 2009)]. Different classes of brain cells show distinctive gene expression patterns (Darmanis et al., 2015 Tasic et al., 2016. Poulin et al. 2016, Mancarci et al. 2017), and scRNA-seq is thus regarded as the most promising technology for accurately resolving cell specificity (Yu and Lin 2016). However, scRNA-seq is difficult to scale to brain-wide analyses, and current brain-wide atlases of gene expression have relied on microarray or ISH. ISH has high spatial resolution, allowing gene expression to be measured in a tissue section with relatively high sensitivity and specificity, but requires a very large number of samples to quantify expression levels across thousands of genes (Unger) et al. 2010). ISH has therefore only been used to construct atlases for species with high tissue availability, such as the mouse (Lein et al. 2007). Microarray, on the other hand, allows the quantification of expression levels of thousands of genes at once by measuring the hybridisation of cRNA (Cy3-labeled RNA) in a tissue sample to particular spot (probe) on the microarray chip. The technique is limited to known gene sequences and is prone to background noise (Okoniewski and Miller, 2006, Royce et al. 2007), but provides a cost-effective way to measure gene transcription in high-throughput manner. It has been used to produce spatially comprehensive atlases of the human (Kang et al. 2011. Hawrylycz et al. 2012, Miller et al., 2014) and non-human primate brain [NIH Blueprint Non-Human Primate (NHP) Atlas (2009), in conjunction with ISH].

As summarized in Keil et al. (2018), there is a large number of gene expression atlases. Due to their high spatial coverage, the two most used brain-wide expression atlases are the Allen Mouse Brain Atlas (AMBA) (Lein et al. 2007) and the Allen Human Brain Atlas (AHBA) (Hawrylycz et al., 2012), both made freely available by the Allen Institute for Brain Science. The AMBA provides an extensive representation of the expression patterns of 19419 genes across the whole mouse brain, using ISH to quantify brain-wide expression patterns with the cellular resolution at each tissue slice with slices acquired every $200 \mu \mathrm{m}$ (the latter resolution depends on the section). Spatially resolved gene expression data can be further parcellated using anatomical atlases of the mouse brain (Johnson et al., 2010, Furth et al. 2018) to acquire averaged expression values through a hierarchy of brain regions defined at different resolution scales.

The AHBA comprises expression measures for 21245 genes (depending on available annotation data) taken from 3702 spatially distinct post-mortem tissue samples distributed throughout the brains of six human donors (Hawrylycz et al. 2012, 2015). Both atlases have been mapped to stereotaxic space, allowing researchers to link spatial variations in gene expression to the spatial variations of a given neural phenotype measured with structural or functional MRI, or other techniques (Furth et al., 2018). Other gene expression databases include both spatial (Fertuzinhos et al. 2014) and spatio-temporal (Ayoub et al., 2011, Belgard et al. 2011, Colantuoni et al. 2011. Miller et al., 2014) atlases, along with the Allen Developing Mouse Brain Atlas (2008), however most of these lack the spatial coverage of the AMBA and AHBA with only a handful regions being assessed across multiple time points. Some gene expression atlases have also been published for the macaque, using ISH and microarray (Bakken et al. 2016), and C. elegans (Harris et al. 2010). The latter database has been curated from published reports and contains binary entries on around $5 \%$ of the $\sim 20000$ genes in the full worm genome, such that the only information encoded is whether a given gene is expressed or not in a neuron.

Gene expression measures can be influenced by a number of technical and biological factors (Fraser et al. 2005. Berchtold et al., 2008, Kumar et al., 2013, Trabzuni et al., 2013). For example, the AHBA consists of data from six donor brains, each varying in characteristics such as age at death, cause of death, sex, and ethnicity. Therefore, any analysis pooling expression measures across brains should ensure that inter-subject variability has not directly influenced the results. The analysis of gene expression measures often involves important additional processing decisions that are not applied consistently and can impact final results. For example, useful steps in processing raw AHBA data prior to analysis include (i) verifying probe-to-gene annotations; (ii) filtering genes that are not expressed above the background; (iii) selecting a representative probe when more than one probe has been used to assay a single gene; (iv) assigning tissue samples to specific brain regions in the imaging dataset; and (v) normalizing expression measures to account for inter-individual differences and outlying values. Each step requires a number of decisions, and best-practice workflows have not been established yet (Arnatkevičiūtė et al., 2018). Finally, gene expression data often shows a strong spatial autocorrelation, such that gene expression is more tightly coupled between regions that are close to each other compared to those that are spatially distant. This trend has been demonstrated in the mouse (Fulcher and Fornito, 2016), human (Richiardi et al. 2015, Krienen et al. 2016, Pantazatos and Li, 2017, Vértes et al. 2016, Arnatkevičiūtè et al. 2018) and head of C. elegans (Arnatkevičiūtè et al. 2018). In order to demonstrate that a putative association between regional variations in gene expression and a given neural phenotype is evident beyond this distance-dependence, potential biases introduced by the dependence can be addressed using methods ranging from 
simple regression (Fulcher and Fornito, 2016), partial Mantel tests (French and Pavlidis, 2011, Ji et al., 2014, Fakhry et al. 2015) or spatially constrained randomisation procedures [for example, see (Vértes et al. 2016. Burt et al., 2017, Seidlitz et al., 2018, Arnatkevičiūtè et al., 2018)].

Brain-wide gene expression measures can be related to a brain network-level phenotype either at the level of specific brain regions (Myers et al. 2007, Rittman et al. 2016, Vértes et al., 2016, Parkes et al., 2017) or using inter-regional transcriptional coupling (Richiardi et al., 2015. Fulcher and Fornito, 2016, Arnatkevičiūté et al., 2018, Romero-Garcia et al. 2018). Analyses of regional gene expression focus on understanding how the expression of a given gene varies across regions, and whether this variation tracks spatial variations in some other phenotype (e.g., regional gray matter volume, or number of connections). In analyses of inter-regional transcriptional coupling or correlated gene expression (CGE), each region's transcriptional profile is mapped as a vector of expression values across all genes, and these vectors are correlated between different regions, thus resulting in a region $\times$ region CGE matrix indicating the similarity between brain regions in terms of their gene expression patterns. Gene-gene co-expression (Eising et al., 2016, Keo et al. 2017, Negi and Guda, 2017), on the other hand, is estimated at the levels of genes (rather than regions). Each gene's expression profile across regions is summarized as a vector, and these vectors are correlated between pairs of genes, resulting in a gene $\times$ gene coexpression matrix demonstrating whether regional expression patterns for gene pairs match. (Note that the term gene coexpression is sometimes used in reference to CGE. We use the current nomenclature to avoid confusion between the two.)

Once a relationship between gene expression and a given neural phenotype has been established, functional groups of genes involved in driving the effect can be identified using gene set enrichment analyses (GSEA) (Subramanian et al., 2005, Irizarry et al., 2009). Since such analyses are often performed across many thousands of genes, GSEA offers a method for determining whether certain categories of genes-e.g., defined by gene ontology (GO) (Ashburner et al. 2000) or KEGG ontology (KO) (Kanehisa and Goto, 2000) — are over-represented in the set of genes showing the strongest associations. This approach allows for a functional interpretation of the results, at the expense of specificity at the level of single genes (i.e., inferences are made about functional groups of genes).

\section{Hubs in brain networks}

Complex behaviours require the coordination and integration of information both within and across different, functionally specialized brain regions. In primate brains, it has long been assumed that association areas, sitting atop the cortical hierarchy, and in interaction with subcortical regions, play an important role in these integrative processes (Felleman and Van Essen, 1991. Mesulam, 1998, Meyer and Damasio, 2009). Structural connectivity studies have confirmed that association areas, and regions of basal ganglia and thalamus, have high levels of connectivity, marking them as network hubs (van den Heuvel and Sporns, 2011). Artificially lesioning these nodes rapidly fragments the network, indicating that they play a vital role in network integration (Albert et al. 2000, van den Heuvel and Sporns, 2011).

Network hubs, the core elements in the network, can be defined using a range of different measures. These measures distinct aspects of topological centrality, which can be defined as the capacity of a node to influence or be influenced by other nodes by virtue of its connection topology (Fornito et al., 2016). The simplest such measure is node degree, which is defined as the number of connections attached to a node. Other commonly used measures include closeness and betweenness centrality, which are both built on the premise that information in the network propagates through the most efficient route (the shortest path between regions), and thus, the centrality of any given node can be quantified by its average shortest path length (closeness), or the number of shortest paths between other nodes on which it lies (betweenness). These measures are often positively correlated across most networks, including the brain, and it is common to find a subset of nodes that score highly on most centrality measures, representing a topologically central network core (Oldham et al., 2018).

Another way to define hubs is in relation to the modular organization of the network. Nodes within a module are densely interconnected with each other and relatively sparsely connected to nodes in other modules. Given a partition of a network into modules [e.g., (Blondel et al. 2008)], the integrative role of a node in the network can be characterized using the participation coefficient: a measure of connection diversity that assigns a high score to nodes with connections distributed evenly across modules. Thus, hubs defined based on the degree centrality can be further classified into 'local hubs', which connect primarily to nodes in the same module (high degree and low participation), and 'connector hubs', which connect to nodes from other modules (Figure 1) (Guimerá et al., 2012).

The interpretation of different measures of network centrality must be moderated by an appreciation of how the network has been constructed. If one investigates structural connectivity (e.g., through electron microscopy, tract tracing, 


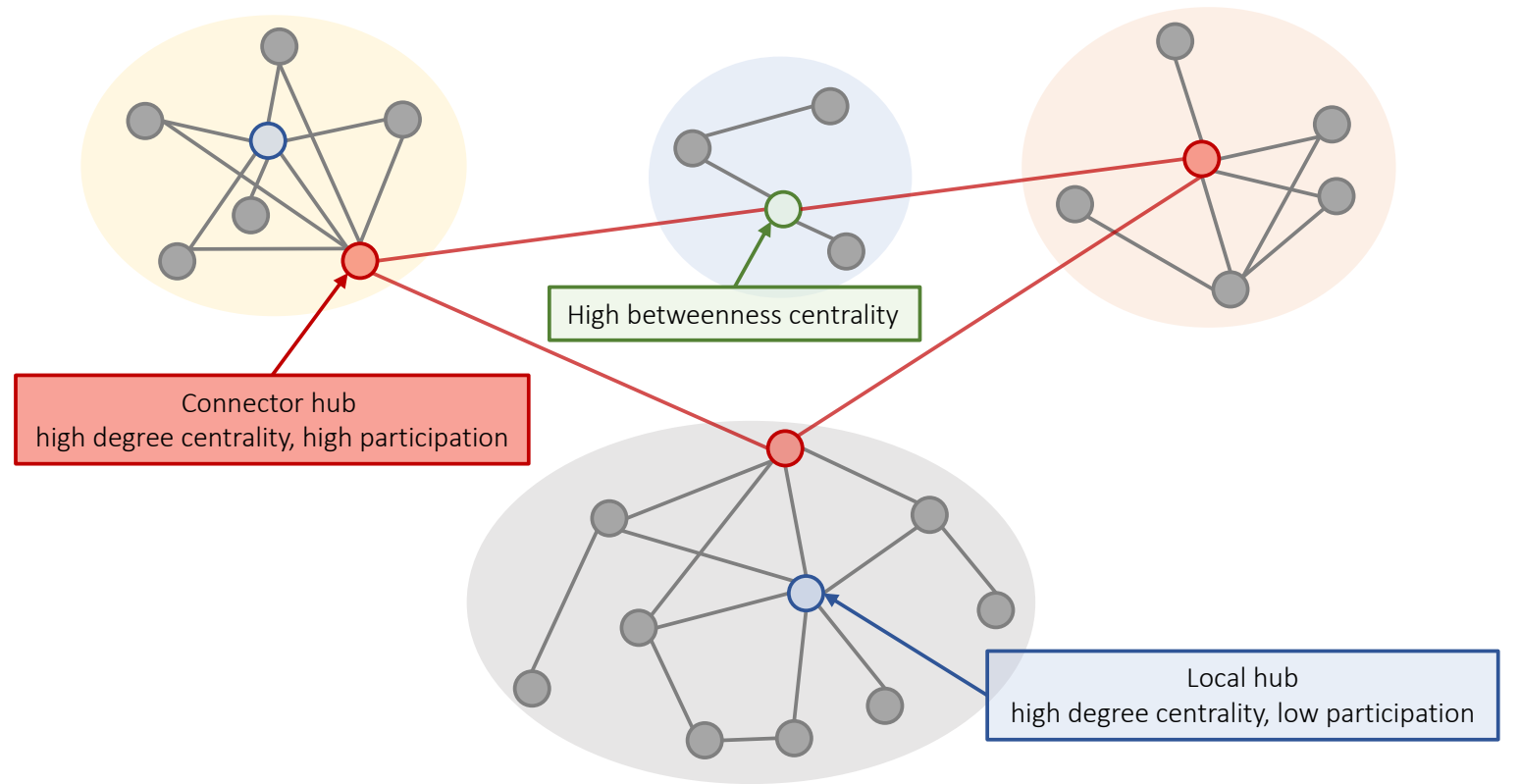

Fig 1. Different concepts of hubness in brain networks. A schematic representation of a modular network where nodes within a module (different background colours) show a relatively high degree of intra-modular connectivity and a low degree of inter-modular connectivity. High degree nodes can be classified into (i) local hubs (blue) that have a high degree centrality and low participation coefficient; and (ii) connector hubs (red) that have high degree and connect to nodes in other modules. Nodes with high betweenness centrality are located on shortest paths between nodes and can play an important role in linking different nodes, even if they have low degree (e.g., the green node supports communication between the yellow and orange modules).

or diffusion MRI) then network edges represent physical connections between network elements, and interpretation is straightforward. If one investigates functional connectivity (e.g., through electrophysiology, calcium imaging, or functional MRI), which captures statistical dependencies between physiological signals recorded at each node (Friston, 1994), the interpretation is less clear and some measures of dependence, such as the correlation coefficient, can bias the topology of the network (Power et al., 2011, Zalesky et al. 2012). Furthermore, different centrality measures make assumptions about how dynamics unfold on the network structure. For example, closeness and betweenness assume information is routed along shortest paths, which may not be a realistic model of communication in nervous systems (Goñi et al. 2014, Mišić et al. 2015, Seguin et al., 2018).

Brain network hubs are densely interconnected, forming a rich-club (Colizza et al., 2006). This property has been observed in the macroscale human connectome (van den Heuvel and Sporns. 2011), the mesoscale connectomes of the mouse (Fulcher and Fornito, 2016), rat (van den Heuvel et al., 2016b), cat (de Reus and van den Heuvel, 2013) and macaque (Harriger et al. 2012), and the micro-scale neuronal connectome of the C.elegans (Towlson et al., 2013) (Figure 2). Given that hubs are distributed throughout the brain and involved in diverse functional systems (de Reus and van den Heuvel, 2013, van den Heuvel and Sporns, 2013, Fulcher and Fornito, 2016), dense inter-connectivity of hub nodes is thought to support efficient integration of different functionally specialized systems (van den Heuvel et al. 2012), and to increase the diversity of the brain's functional repertoire (Senden et al., 2014). This integrative capacity comes at cost, with connections between hubs extending over longer anatomical distances than other types of connections (van den Heuvel and Sporns, 2011, Harriger et al., 2012, Fulcher and Fornito, 2016, Arnatkevičiūtè et al., 2018). Hub regions also have the highest levels of resting metabolism (Vaishnavi et al., 2010, Tomasi et al., 2013) and blood flow (Liang et al. 2013). This high metabolic cost is thought to partly explain why pathology preferentially accumulates in brain network hubs across a wide range of diverse neurological diseases (Bullmore and Sporns, 2012. Crossley et al. 2014, Fornito et al., 2015).

The mechanisms resulting in the emergence of network hubs are unknown, but geometric constraints and evolutionary pressures to maximise adaptive function may play a role (Henderson and Robinson, 2014, Roberts et al. 


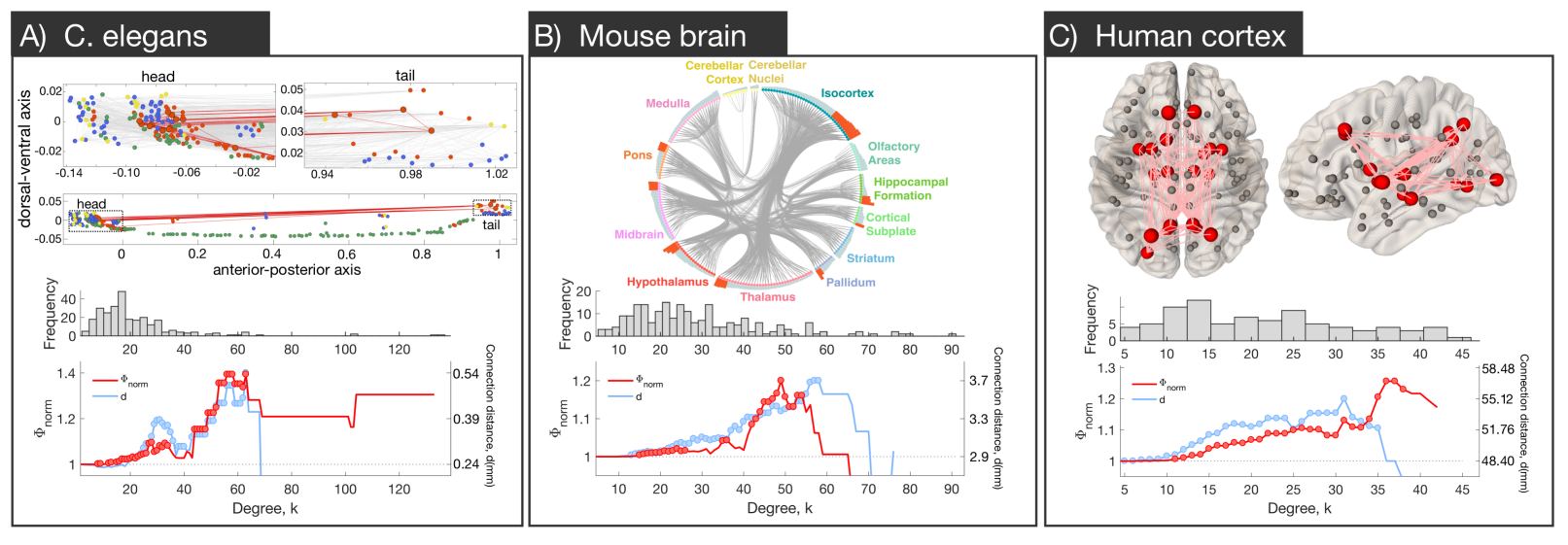

Fig 2. Rich club connectivity in different species. Top row: the spatial location of hubs in C. elegans (A), mouse (B), and human (C). (A) Neurons are represented as nodes with colours corresponding to neuron type: interneurons (red), motor neurons (green), sensory neurons (blue), multimodal neurons (yellow). Hub neurons (neurons with node degree, denoted $k$, greater than 44) are shown as circles outlined in black. Connections between hubs are shown in red; other connections shown in gray in the upper plots. The upper part represents zoomed-in plots of the head and tail that are shown as dotted rectangles in the lower plot [adapted and reproduced from Arnatkevičiūtė et al. (2018)].

(B) Meso-scale connectome of the mouse. Hub regions (regions with $k>44$ ) are distributed across the whole brain and contain areas in isocortex, striatum, hippocampal formation, pallidum, thalamus, hypothalamus, midbrain, pons, and cortical subplate [adapted and reproduced from Fulcher and Fornito (2016)]. (C) Macro-scale connectome of the human brain. Hub regions (regions with $k>30$ ) are shown as big red spheres while other regions as smaller gray spheres. Connections between hubs are shown in pink. Hubs are bilateral: lingual gyrus, precuneus, superior frontal gyrus, superior parietal gyrus, insula, thalamus, putamen and hippocampus; right pallidum; left caudate and lateral occipital gyrus. Middle row: distribution of degree values across nodes. In each network, the distribution is heavy-tailed, consistent with the presence of highly connected hub nodes. Bottom row: Normalised rich club coefficient $\Phi_{\text {norm }}$ (red) and average connection distance of hub-hub links, $d$ (blue), as a function of degree $(k)$ at which hubs are defined. The coefficient $\Phi_{\text {norm }}$ is defined by thresholding the network at a given level of $k$, calculating the density of connections between hub nodes (all nodes with degree $>k$ ), and normalizing this value by the corresponding value obtained in an ensemble of appropriately matched surrogate graphs. The normalized coefficient therefore quantifies the degree to which the density of connections between hubs exceeds chance expectations. Since the threshold to define hubs is arbitrary, the coefficient is evaluated across all possible values of $k$. A rise in $\Phi_{\text {norm }}$ at high levels of $k$ is consistent with rich-club organization. Red circles indicate $\Phi_{\text {norm }}$ values that are significantly higher than an ensemble of 10000 null networks (permutation test $p<0.05$ ). Blue circles indicate where the mean connection distance between hubs is significantly greater relative to other links in the network (one-sided Welch's t-test; $p<0.05$ ).

2016, Betzel and Bassett, 2017). More generally, the striking conservation of rich-club organization across species and scales suggests that genetic influences may also play a prominent role. We now turn our attention to recent studies investigating the transcriptional correlates of hub connectivity by integrating connectomic data with spatially comprehensive gene expression databases across different species and scales.

\section{The molecular correlates of hub connectivity}

The first study to link transcriptional measures to the hub connectivity (Rubinov et al. 2015) combined gene expression data from the AMBA (Lein et al., 2007) with a mouse connectome inferred statistically from 461 tract-tracing studies (Oh et al. 2014). Data from these anterograde tracer injections into the right hemisphere were aggregated into a directed and weighted connectivity matrix comprising of 112 bilaterally symmetrical cortical and subcortical nodes defining edge weights as normalised connection densities and ranging over four orders of magnitude, with $53 \%$ of all possible pairs of regions showing some level of non-zero connectivty. The authors identified a subset of nodes with high degree and a high participation coefficient, indicating that they were highly connected while also being connected 
to nodes in diverse functional systems. Using partial least squares (PLS) (Hervé, 2010), they were able to derive a linear combination of genes whose expression levels explained $48 \%$ of the variance in nodal particiation coefficient. The analysis focused on a subset of 3380 genes form the AMBA that passed quality control criteria and were assayed in at least one additional independent experiment allowing the authors to evaluate gene expression reproducibility. The genes weighting strongly on the participation-related component were enriched for GO categories such as learning, cognition, and memory, suggesting a link between the expression of genes related to regional variations in network participation and those implicated in cognition.

In a subsequent analysis of the Allen Institute mouse connectome, Fulcher and Fornito (2016) used a parcellation comprising 213 regions linked by 3063 connections (6.9\% of all possible links), focusing on the right hemisphere only (where complete information on afferent and efferent connectivity was available), in combination with ISH measures of expression across 17642 genes in the AMBA (Lein et al. 2007). Their primary aim was to characterize how coupled patterns of gene expression between regions (i.e., correlated gene expression or CGE) relate to network topology. After confirming that the right hemisphere of the mouse connectome did indeed show evidence of rich-club organization, and that connections between hubs were both the most costly (measured by connection distance, reciprocity and weight) and central (measured using edge betweenness centrality and an alternative measure called communicability, that does rely on shortest path communication) connections of the network, they distinguished between three topological classes of connections following the work of van den Heuvel et al. (2012): (i) rich links, which connect two hubs (where hub is defined based on degree); (ii) feeder links, which connect a hub to non-hub (feed-out) or a non-hub to a hub (feed-in); and (iii) peripheral links, which connect two non-hubs (Figure $3 \mathrm{~A}$ ). Across a wide range of thresholds for defining a hub, CGE was highest for rich links, followed by feeder, and lowest for peripheral edges, with CGE showing a sharp rise at a hub threshold range that coincided with a regime in which a significant topological rich club was observed (Figure $3 \mathrm{~B}$ ). This tightly coupled transcriptional activity between hub nodes defied a general trend in the brain where CGE between two areas decayed sharply (exponentially) as a function of their distance. That is, despite connected hubs being separated by longer anatomical distances than other pairs of regions, they showed the highest levels of transcriptional coupling (note that CGE measures were corrected for this dependence). Enrichment analysis showed that this effect was driven by genes regulating the oxidative synthesis and metabolism of ATP - the primary energetic source of neuronal communication. By comparison, an enrichment analysis comparing connected to unconnected regions (regardless of whether those connections involved hubs) found significant involvement of a large number of GO categories related to synaptic plasticity and communication, axon structure, and metabolism. These findings suggest that while genes involved in forming and maintaining synapses and axons are important for establishing a connection between two regions, the primary genomic distinction between different topological classes of connections (as defined in relation to hubs) is related to the metabolic requirements of those connections.

More recently, we found a qualitatively similar pattern of elevated CGE in rich links in the nematode C. elegans connectome (Arnatkevičiūtè et al. 2018). Combining electron micrograph data defining the electrochemical connectome of 279 neurons (Varshney et al. 2011) with binary gene expression profiles across 948 genes (Figure $3 \mathrm{C}$ ) acquired from WormBase (Harris et al. 2010), we identified the same trend for CGE to be highest for rich links, followed by feeder, and then peripheral edges (Figure 3D). The involvement of metabolic genes in rich-club connectivity-as in the mesoscopic mouse connectome (Fulcher and Fornito, 2016) — could not be confirmed due to limited gene expression data in the worm, but analysis of the available data indicated that glutamate signalling and neuronal communication genes made the strongest contribution to elevated CGE for hub-hub connections (Arnatkevičiūte et al. 2018). Leveraging the extensive additional data on neuronal phenotypes available for the worm, we found that elevated CGE for connected hubs could not be explained by a range of other properties such as neuronal lineage distance (number of cell divisions separating pairs of neurons from a common ancestor), differences in birth time, neuronal subtype (sensory, motor, or interneuron), chemically secreted neurotransmitter, anatomical separation distance or topological module affiliation. However, the effect did seem to be driven by the fact that most hubs in the worm connectome are command interneurons, a specialized class of neurons that regulates motion. Motion is one of the more complex behaviors in the worm's repertoire, and these findings parallel evidence in primates that network hubs are primarily located in association cortices, which are thought to mediate higher-order cognition (Achard et al., 2006. Sporns et al., 2007). Thus, despite numerous differences in the data, including different gene annotation methods ( $\sim 20000$ ISH genes in mouse versus $\sim 1000$ binary literature-curated annotations in worm), the type of the neural system (spatially continuous macroscopic mouse brain $v s$ spatially separated C. elegans nervous system), and the orders of magnitude differences in scale, both studies demonstrated the same general pattern of increased transcriptional similarity across topologically central hub nodes. 

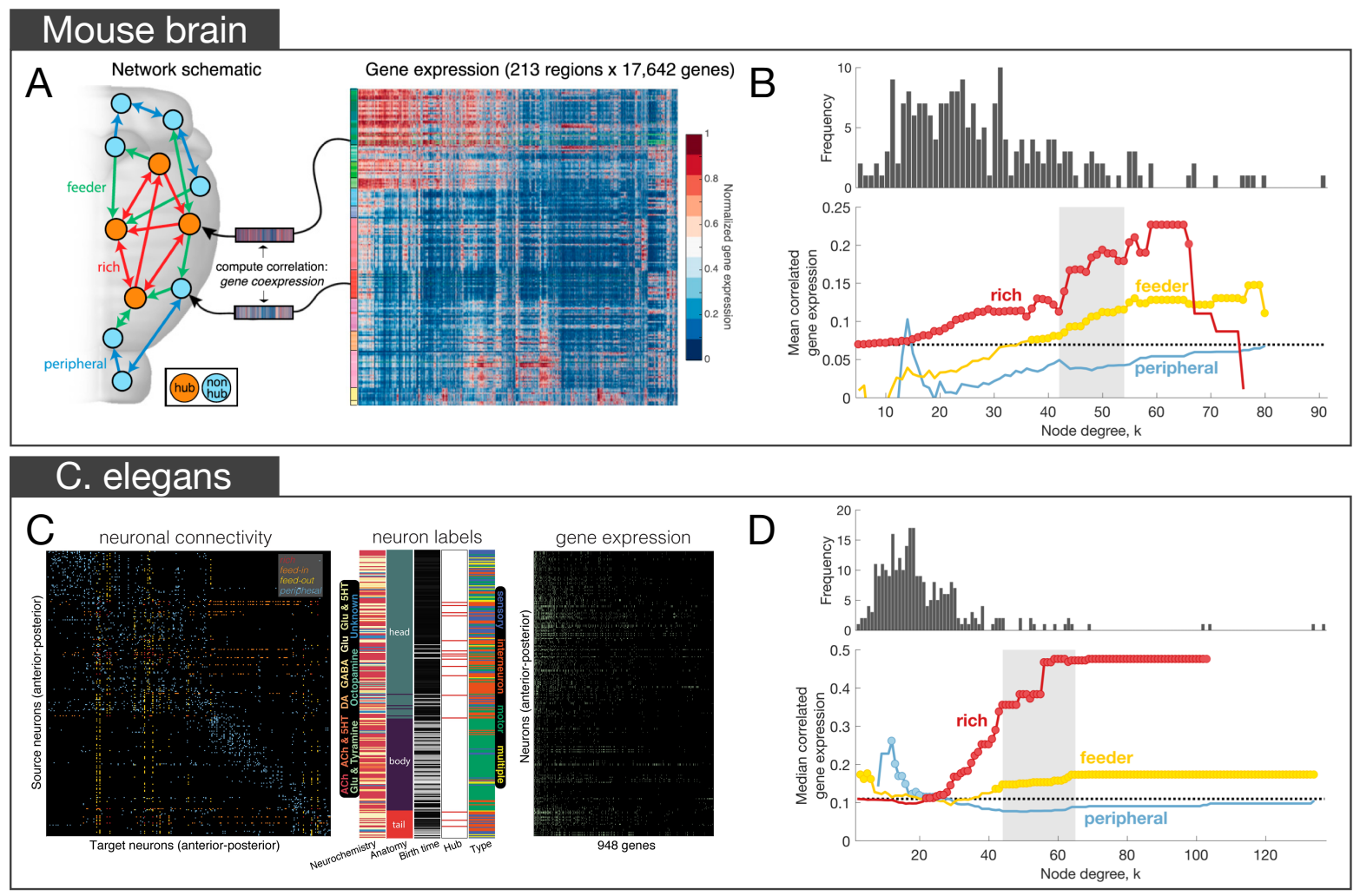

Fig 3. Empirical studies investigating the transcriptional properties of hub connectivity in mouse (A,B) and C. elegans (C,D). (A) The schematic representation of different types of connections in the mouse brain: rich (connecting a hub to a hub)—red, feeder (connecting a hub to a non-hub or a non-hub to a hub)_-green, peripheral (connecting a non-hub to a non-hub)_blue. Links in the connectome were categorized across this scheme. For each region, a vector of gene expression values was extracted as the corresponding row of the region in the full gene expression matrix comprising the AMBA. The matrix represents the normalised gene expression of 17642 genes (columns) across 213 regions (rows). Gene expression profiles for each region were then used to estimate correlated gene expression (CGE) between region pairs. (B) Mean correlated gene expression for rich, feeder and peripheral links as a function of node degree $(k)$ where hubs are nodes with degree $>k$. The mean CGE of rich links increases at levels of $k$ that coincide with a regime where evidence of topological rich-club organization is found indicating that CGE is highest for connected pairs of network hubs. The topological rich club regime (determined from the network topology, see Figure 2A) shaded gray. Circles indicate a statistically significant increase in correlated gene expression for a given link type relative to the rest of the network (one-sided Welch-s t-test; $p<0.05$ ) [adapted and reproduced from Fulcher and Fornito (2016)]; (C) Neuron-and-synapse connectome of $C$. elegans, reconstructed for 279 neurons using electron microscopy. Connections coloured according to how they connect hubs (neurons with degree $>44$ ) and non-hubs (neurons with degree $\leq 44$ ): red (rich links connecting hubs), orange (feed-in links connecting a non-hub to a hub), yellow (feed-out links connecting a hub to a non-hub), blue (peripheral links connecting non-hubs). Middle: additional data acquired for each neuron such as its: chemically secreted transmitter, anatomical location, birth time, hub status and neuronal type. Right binary gene expression profile for each of the 279 neurons (rows) across 948 genes (columns). (D) Median CGE for each connection type (feed-in and feed-out connections are combined and represented as feeder) as a function of node degree $k$. The topological rich club regime (determined from the network topology, see Figure 2 A) shaded gray. Circles indicate a statistically significant increase in CGE in a given link type relative to the rest of the network (one-sided Wilcoxon rank sum test, $p<0.05$ ) [adapted and reproduced from Arnatkevičiūtè et al. (2018)].

In light of the findings in both mouse and C.elegans, where several groups of genes implicated in cognition (Rubinov et al. 2015), oxidative metabolism (Fulcher and Fornito, 2016), and neuronal communication (Arnatkevičiūtè 
et al. 2018) have been identified as being related to hub connectivity, one could wonder whether the same genes are involved in the hub connectivity of the human brain. The first analysis to link gene expression and hub connectivity in humans was performed by Vértes et al. (2016), who combined resting-state fMRI (rs-fMRI) data with the high coverage genome-wide gene expression from AHBA (Hawrylycz et al. 2012). Rendering rs-fMRI data for 285 cortical regions as a binary undirected network, thresholded to retain $10 \%$ of all possible connections, they measured three different properties of each node: its within-module connectivity, its participation coefficient (between-module connectivity), and its average Euclidean distance from other nodes. PLS identified three components that collectively accounted for $37 \%$ of the total variance in nodal metrics with the first component exhibiting a positive correlation with intra-modular degree and a negative correlation with average nodal distance, corresponding to high degree nodes that mostly form short-range within-module connections. Genes positively loading on this component were enriched for GO categories related to transcriptional regulation. The second component was positively related to both the participation coefficient and average nodal distance, thus representing nodes with long connections that extend between modules, consistent with the integrative hubs of the network (Figure $4 \mathrm{~A}$ ). As seen in the analysis of the structural connectivity analysis of the mouse (Fulcher and Fornito, 2016), genes loading positively on this component were enriched in GO categories related to oxidative metabolism and mitochondrial function. These genes also showed significant overrepresentation for a set of 19 genes (Krienen et al. 2016) selectively enriched in the supragranular layers of the human cortex (HSE human supragranular enriched genes) with some of those genes being implicated in the formation of corticocortical projections emanating from the higher layers of the cortex (Krienen et al., 2016). Together these findings suggest that hubs across species demonstrate conserved transcriptional properties related to their high metabolic demands.

\section{Inter-modular hubs}

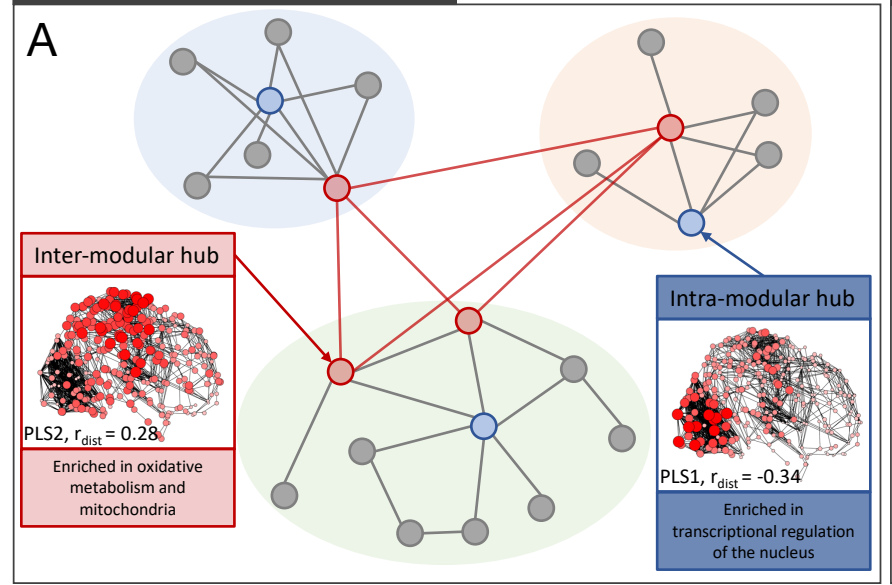

\section{Cortical consolidation}

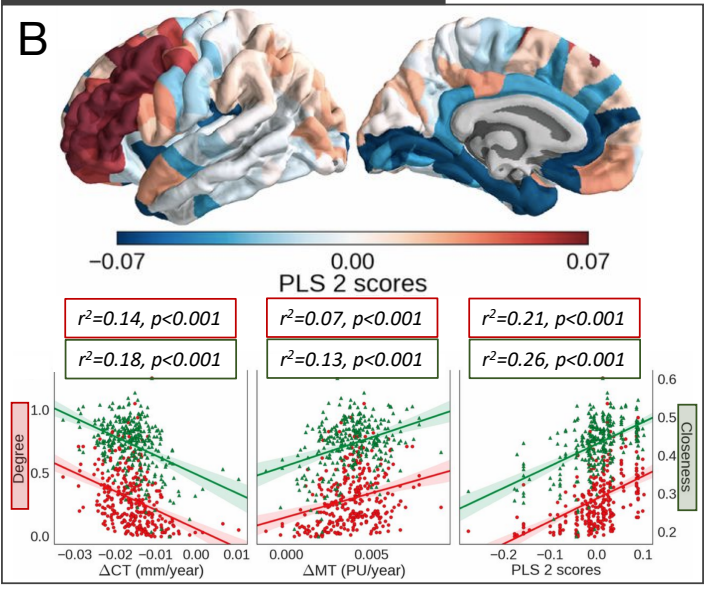

Fig 4. Empirical studies investigating the transcriptional properties of hub connectivity in human. (A) A schematic representation of the modular organization of the connectome demonstrating the key properties of inter- and intramodular hubs based on Vértes et al. (2016). Intra-modular hubs (blue nodes) mostly connect nodes within the same module and are characterized with a relatively short connection distances; characterized by the PLS1. Intra-modular hubs (red nodes) have a more diverse connectivity profile with connections extending long distances and connecting nodes from different modules; characterized by the PLS2. Size and color saturation of the nodes in the connectome corresponds to the regional scores on PLS1 (Intra-modular hub) and PLS2 (Inter-modular hub) to represent the spatial pattern of transcriptional profiles [adapted and modified from/Vértes et al. (2016)]. (B) Gene expression and cortical consolidation in adolescence based on Whitaker et al. (2016), top: spatial topography of the second component from a PLS analysis corresponding to cortical consolidation during adolescence, defined as cortical shrinkage/myelination. Genes identified in this profile are related to synaptic transmission and risk to schizophrenia, among others, and are overexpressed in prefrontal areas of the cortex; bottom: hubs in the structural covariance network experience faster rates of cortical thinning (CT) and myelination. The PLS2 gene expression profile is also significantly associated with degree, meaning that hubs are likely to over-express those genes [adapted and modified from Whitaker et al. (2016)].

It is well-known that the human brain undergoes an extended period of development during adolescence that is critical for brain maturation and coincides with the period of peak risk for many mental disorders (Paus et al. 
2008). Some of those developmental changes particularly target hub regions (Dennis et al., 2013, Hwang et al. 2013. Baker et al., 2015) [for a review see (Cao et al., 2016)]. Whitaker et al. (2016) examined a large sample of adolescents (279, aged 14 to 24 years old) and found that topologically central hubs of the cortical structural covariance networks undergo an increased rate of consolidation, defined by increased cortical thinning and enhanced myelination (Figure 4B). Components of transcriptional variance that correlated with this consolidation were extracted using PLS, employing the full set of 20737 genes from the AHBA. The first two components explaining $28 \%$ of the variance in MRI measures were related to the baseline measures of cortical thickness and myelination (PLS1), and cortical shrinkage and myelination - consolidation over time (PLS2) (Figure43) respectively. The PLS2 component involved contributions from genes regulating synaptic transmission and a set of genes linked to risk for schizophrenia, suggesting that deviation from the normal developmental consolidation of hub regions might manifest as an intermediate phenotype for schizophrenia (Whitaker et al. 2016), consistent with evidence that hubs are disproportionately impacted by the disease (Van Den Heuvel et al., 2013, Crossley et al., 2014, Klauser et al., 2016) and that regional variations in the expression of schizophrenia risk genes track the regional variations in the magnitude of group differences in connectivity between controls and patients (Romme et al. 2017).

Importantly, this work implies that genes involved in the development of hubs, which relate to myelination and synaptic transmission, are distinct from those implicated in cross-sectional studies of adult hub connectivity, which implicate metabolic genes. In other words, the genetic mechanisms underlying the development of hub connectivity may differ from those involved in sustaining the functional role that hubs play in a mature neuronal system. The further development of brain-wide atlases of developmental changes in gene expression will help shed light on how such differences can be leveraged to gain insight into the development of different brain disorders.

\section{Conclusions and further directions}

Brain-wide gene expression atlases provide exciting opportunities to link different scales of brain organisation. At the same time, integrating such data with connectomic measures poses challenges. Given the nascense of this field, no standardized data processing pipelines have been developed, with widespread inconsistencies in processing of the same transcriptional data across studies (Arnatkevičiūtè et al. 2018) complicating direct comparison between findings, even within the same species. Nonetheless, the available studies-conducted in diverse species and using different measures of brain connectivity and gene expression acquired at different resolution scales-point to a conserved transcriptional signature of hub connectivity related to genes regulating neuronal communication and metabolism, consistent with the high centrality and metabolic cost of hub regions (Bullmore and Sporns, 2009).

One limitation affecting the human data is that the gene expression measures are derived from bulk tissue samples. The cellular composition of these samples can influence measured gene expression patterns, such that two samples can differ in their transcriptional properties simply due to the differences in the density of distinct cell types. Single-cell transcriptomics is able to provide precise gene expression measurements in individual cells, thus resolving cell-specific transcriptional profiles. While scRNA-seq is not currently feasible for the whole human brain, the expression profiles of specific cell groups in the adult (Johnson et al. 2015, Hu and Wang, 2017, Picardi et al., 2017) and developing brain (Zhong et al., 2018) are being characterized.

These limitations notwithstanding, the consistency of results considered here-often identified through unbiased, data-driven techniques - demonstrate the potential utility of brain-wide transcriptomic measures in yielding biologically meaningful insights to otherwise abstract graph-theoretical structures such as hubs and other neural phenotypes. With the availability of new resources and developments in neuroimaging, the combination of such data across resolution scales offers a promising way forward for uncovering the molecular mechanisms that drive the large-scale organization of the connectome.

\section{Conflict of Interest Statement}

The authors declare that the research was conducted in the absence of any commercial or financial relationships that could be construed as a potential conflict of interest. 


\section{Author Contributions}

AA Wrote and edited the manuscript; BDF Provided feedback, structured and edited the manuscript; AF Provided feedback, structured and edited the manuscript; All the authors planned the structure of the manuscript.

\section{Funding}

AF was supported by the Australian Research Council (ID: FT130100589) and National Health and Medical Research council (IDs: 1146292). BDF is supported by an NHMRC Early Career Fellowship (ID: 1089718).

\section{Acknowledgments}

We would like to thank Stuart Oldham for processing human DWI data used in Figure 2

\section{References}

Achard, S., Salvador, R., Whitcher, B., Suckling, J., Bullmore, E., 2006. A resilient, low-frequency, small-world human brain functional network with highly connected association cortical hubs. J. Neurosci. 26, 63-72. doi:10.1523/ JNEUROSCI.3874-05.2006

Albert, R., Jeong, H., Barabási, A.L., 2000. Error and attack tolerance of complex networks. Nature 406, 378-382. doi: $10.1038 / 35019019$.

Anderson, K.M., Krienen, F.M., Choi, E.Y., Reinen, J.M., Yeo, B.T.T., Holmes, A.J., 2018. Gene expression links functional networks across cortex and striatum. Nat. Commun. 9, 1428. doi:10.1038/s41467-018-03811-x.

Arnatkevičiūtė, A., Fulcher, B.D., Pocock, R., Fornito, A., 2018. Hub connectivity, neuronal diversity, and gene expression in the Caenorhabditis elegans connectome. PLoS Comput. Biol. 14, e1005989. doi 10.1371 / journal. pcbi.1005989.

Arnatkevičiūtè, A., Fulcher, B.D., Fornito, A., 2018. A practical guide to linking brain-wide gene expression and neuroimaging data. bioRxiv doi:10.1101/380089.

Ashburner, M., Ball, C.A., Blake, J.A., Botstein, D., Butler, H., et al., 2000. Gene Ontology: tool for the unification of biology. Nat. Genet. 25, 25-29. doi:10.1038/75556.

Ayoub, A.E., Oh, S., Xie, Y., Leng, J., Cotney, J., Dominguez, M.H., Noonan, J.P., Rakic, P., 2011. Transcriptional programs in transient embryonic zones of the cerebral cortex defined by high-resolution mRNA sequencing. Proc. Natl. Acad. Sci. 108, 14950-14955. doi:10.1073/pnas.1112213108.

Baker, S.T.E., Lubman, D.I., Yücel, M., Allen, N.B., Whittle, S., Fulcher, B.D., Zalesky, A., Fornito, A., Yucel, M., Allen, N.B., Whittle, S., Fulcher, B.D., Zalesky, A., Fornito, A., 2015. Developmental changes in brain network hub connectivity in late adolescence. J. Neurosci. 35, 9078-9087. doi 10.1523/jneurosci.5043-14.2015.

Bakken, T.E., Miller, J.A., Ding, S.L., Sunkin, S.M., Smith, K.A., et al. 2016. A comprehensive transcriptional map of primate brain development. Nature 535, 367-375. doi $10.1038 /$ nature18637.

Baruch, L., Itzkovitz, S., Golan-Mashiach, M., Shapiro, E., Segal, E., 2008. Using expression profiles of Caenorhabditis elegans neurons to identify genes that mediate synaptic connectivity. PLoS Comput. Biol. 4, 1-10. doi:10.1371/ journal.pcbi.1000120.

Bassett, D.S., Bullmore, E.T., 2009. Human brain networks in health and disease. Curr. Opin. Neurol. 22, 340-347. doi $10.1097 / \mathrm{WCO} .0 \mathrm{~b} 013 \mathrm{e} 32832 \mathrm{~d} 93 \mathrm{dd}$.

Bassett, D.S., Bullmore, E.T., 2017. Small-world brain networks revisited. Neurosci. 23, 499-516. doi $10.1177 /$ 1073858416667720 . 
Bassett, D.S., Greenfield, D.L., Meyer-Lindenberg, A., Weinberger, D.R., Moore, S.W., Bullmore, E.T., 2010. Efficient physical embedding of topologically complex information processing networks in brains and computer circuits. PLoS Comput. Biol. 6, e1000748. doi:10.1371/journal.pcbi.1000748

Belgard, T., Marques, A., Oliver, P., Abaan, H.O., Sirey, T., Hoerder-Suabedissen, A., García-Moreno, F., Molnár, Z., Margulies, E., Ponting, C., 2011. A transcriptomic atlas of mouse neocortical layers. Neuron 71, 605-616. doi $10.1016 / j$. neuron.2011.06.039.

Berchtold, N.C., Cribbs, D.H., Coleman, P.D., Rogers, J., Head, E., Kim, R., Beach, T., Miller, C., Troncoso, J., Trojanowski, J.Q., Zielke, H.R., Cotman, C.W., 2008. Gene expression changes in the course of normal brain aging are sexually dimorphic. Proc. Natl. Acad. Sci. 105, 15605-10. doi $10.1073 /$ pnas.0806883105.

Betzel, R.F., Bassett, D.S., 2017. Multi-scale brain networks. Neuroimage 160, 73-83. doi:10.1016/J. NEUROIMAGE.2016.11.006.

Blondel, V.D., Guillaume, J.L., Lambiotte, R., Lefebvre, E., 2008. Fast unfolding of communities in large networks. J. Stat. Mech. Theory Exp. 2008, P10008. doi:10.1088/1742-5468/2008/10/P10008.

Bohlken, M.M., Mandl, R.C., Brouwer, R.M., van den Heuvel, M.P., Hedman, A.M., Kahn, R.S., Hulshoff Pol, H.E., 2014. Heritability of structural brain network topology: A DTI study of 156 twins. Hum. Brain Mapp. 35, 5295-5305. doi $10.1002 / \mathrm{hbm} .22550$.

Brown, J.A., Terashima, K.H., Burggren, A.C., Ercoli, L.M., Miller, K.J., Small, G.W., Bookheimer, S.Y., 2011. Brain network local interconnectivity loss in aging APOE-4 allele carriers. Proc. Natl. Acad. Sci. 108, 20760-20765. doi $10.1073 /$ pnas.1109038108.

Bullmore, E., Sporns, O., 2009. Complex brain networks: graph theoretical analysis of structural and functional systems. Nat. Rev. Neurosci. 10, 186-198. doi:10.1038/nrn2575.

Bullmore, E., Sporns, O., 2012. The economy of brain network organization. Nat. Rev. Neurosci. 13, 336-49. doi $10.1038 / \mathrm{nrn} 3214$.

Burt, J.B., Demirtas, M., Eckner, W.J., Navejar, N.M., Ji, J.L., Martin, W.J., Bernacchia, A., Anticevic, A., Murray, J.D., 2017. Hierarchy of transcriptomic specialization across human cortex captured by myelin map topography. doi:10.1101/199703.

Buzsáki, G., Draguhn, A., 2004. Neuronal oscillations in cortical networks. Science 304, 1926-9. doi $10.1126 /$ science.1099745.

Cao, M., Huang, H., Peng, Y., Dong, Q., He, Y., 2016. Toward developmental connectomics of the human brain. Front. Neuroanat. 10, 25-. doi $10.3389 /$ fnana.2016.00025.

Cioli, C., Abdi, H., Beaton, D., Burnod, Y., Mesmoudi, S., 2014. Differences in human cortical gene expression match the temporal properties of large-scale functional networks. PLoS One 9, e115913. doi $10.1371 /$ journal. pone.0115913.

Colantuoni, C., Lipska, B.K., Ye, T., Hyde, T.M., Tao, R., Leek, J.T., Colantuoni, E.A., Elkahloun, A.G., Herman, M.M., Weinberger, D.R., Kleinman, J.E., 2011. Temporal dynamics and genetic control of transcription in the human prefrontal cortex. Nature 478, 519-23. doi:10.1038/nature10524.

Colclough, G.L., Smith, S.M., Nichols, T.E., Winkler, A.M., Sotiropoulos, S.N., Glasser, M.F., Van Essen, D.C., Woolrich, M.W., 2017. The heritability of multi-modal connectivity in human brain activity. Elife 6, e20178. doi:10.7554/eLife.20178.

Colizza, V., Flammini, A., Serrano, M.A., Vespignani, A., 2006. Detecting rich-club ordering in complex networks. Nat. Phys. 2, 110-115. doi $10.1038 /$ nphys209.

Crossley, N.A., Mechelli, A., Scott, J., Carletti, F., Fox, P.T., McGuire, P., Bullmore, E.T., 2014. The hubs of the human connectome are generally implicated in the anatomy of brain disorders. Brain a J. Neurol. 137, 2382-95. doi:10.1093/brain/awu132. 
Darmanis, S., Sloan, S.A., Zhang, Y., Enge, M., Caneda, C., Shuer, L.M., Hayden Gephart, M.G., Barres, B.A., Quake, S.R., 2015. A survey of human brain transcriptome diversity at the single cell level. Proc. Natl. Acad. Sci. 112, 7285-90. doi:10.1073/pnas.1507125112.

Dennis, E.L., Jahanshad, N., Rudie, J.D., Brown, J.A., Johnson, K., McMahon, K.L., de Zubicaray, G.I., Montgomery, G., Martin, N.G., Wright, M.J., Bookheimer, S.Y., Dapretto, M., Toga, A.W., Thompson, P.M., 2011. Altered structural brain connectivity in healthy carriers of the autism risk gene, CNTNAP2. Brain Connect. 1, 447-459. doi:10.1089/brain.2011.0064.

Dennis, E.L., Jahanshad, N., Toga, A.W., McMahon, K.L., de Zubicaray, G.I., Hickie, I., Wright, M.J., Thompson, P.M., 2013. Development of the rich club in brain connectivity networks from 438 adolescents \& adults aged 12 to 30. Proceedings. IEEE Int. Symp. Biomed. Imaging, 624-627 doi 10.1109 / ISBI . 2013.6556552.

Eising, E., Huisman, S.M., Mahfouz, A., Vijfhuizen, L.S., Anttila, V., et al. , 2016. Gene co-expression analysis identifies brain regions and cell types involved in migraine pathophysiology: a GWAS-based study using the Allen Human Brain Atlas. Hum. Genet. 135, 425-439. doi:10.1007/s00439-016-1638-x.

Fakhry, A., Ji, S., 2015. High-resolution prediction of mouse brain connectivity using gene expression patterns. Methods 73,71-8. doi:10.1016/j.ymeth.2014.07.011.

Fakhry, A., Zeng, T., Peng, H., Ji, S., 2015. Global analysis of gene expression and projection target correlations in the mouse brain. Brain informatics 2, 107-117. doi $10.1007 / \mathrm{s} 40708-015-0014-2$.

Felleman, D.J., Van Essen, D.C., 1991. Distributed hierarchical processing in the primate cerebral cortex. Cereb. Cortex 1, 1-47.

Fertuzinhos, S., Li, M., Kawasawa, Y., Ivic, V., Franjic, D., Singh, D., Crair, M., Šestan, N., 2014. Laminar and temporal expression dynamics of coding and noncoding RNAs in the mouse neocortex. Cell Rep. 6, 938-950. doi:10.1016/j.celrep.2014.01.036.

Forest, M., Iturria-Medina, Y., Goldman, J.S., Kleinman, C.L., Lovato, A., Oros Klein, K., Evans, A., Ciampi, A., Labbe, A., Greenwood, C.M., 2017. Gene networks show associations with seed region connectivity. Hum. Brain Mapp. 38, 3126-3140. doi:10.1002/hbm. 23579

Fornito, A., Zalesky, A., Bassett, D.S., Meunier, D., Ellison-Wright, I., et al. 2011. Genetic influences on cost-efficient organization of human cortical functional networks. J. Neurosci. 31, 3261-70. doi:10.1523/ JNEUROSCI. $4858-10.2011$.

Fornito, A., Zalesky, A., Breakspear, M., 2013. Graph analysis of the human connectome: promise, progress, and pitfalls. Neuroimage 80, 426-444. doi:10.1016/j.neuroimage.2013.04.087.

Fornito, A., Zalesky, A., Breakspear, M., 2015. The connectomics of brain disorders. Nat. Rev. Neurosci. 16, $159-172$. doi:10.1038/nrn3901.

Fornito, A., Zalesky, A., Bullmore, E., 2016. Fundamentals of Brain Network Analysis. Academic Press. doi: 10 . 1016/B978-0-12-407908-3.00011-X.

Fraser, H.B., Khaitovich, P., Plotkin, J.B., Pääbo, S., Eisen, M.B., 2005. Aging and gene expression in the primate brain. PLoS Biol. 3, e274. doi:10.1371/ journal.pbio.0030274

French, L., Pavlidis, P., 2011. Relationships between gene expression and brain wiring in the adult rodent brain. PLoS Comput. Biol. 7, e1001049. doi:10.1371/journal.pcbi.1001049

Friston, K.J., 1994. Functional and effective connectivity in neuroimaging: A synthesis. Hum. Brain Mapp. 2, 56-78. doi: $10.1002 / \mathrm{hbm} .460020107$.

Fulcher, B.D., Fornito, A., 2016. A transcriptional signature of hub connectivity in the mouse connectome. Proc. Natl. Acad. Sci. 113, 1435-40. doi:10.1073/pnas.1513302113. 
Fürth, D., Vaissiére, T., Tzortzi, O., Xuan, Y., Märtin, A., Lazaridis, I., Spigolon, G., Fisone, G., Tomer, R., Deisseroth, K., Carlén, M., Miller, C.A., Rumbaugh, G., Meletis, K., 2018. An interactive framework for whole-brain maps at cellular resolution. Nat. Neurosci. 21, 139-149. doi:10.1038/s41593-017-0027-7.

Goel, P., Kuceyeski, A., LoCastro, E., Raj, A., 2014. Spatial patterns of genome-wide expression profiles reflect anatomic and fiber connectivity architecture of healthy human brain. Hum. Brain Mapp. 35, 4204-4218. doi:10. $1002 / \mathrm{hbm} .22471$

Goñi, J., van den Heuvel, M.P., Avena-Koenigsberger, A., Velez de Mendizabal, N., Betzel, R.F., Griffa, A., Hagmann, P., Corominas-Murtra, B., Thiran, J.P., Sporns, O., 2014. Resting-brain functional connectivity predicted by analytic measures of network communication. Proc. Natl. Acad. Sci. 111,833-8. doi:10.1073/pnas.1315529111.

Gămănuţ, R., Kennedy, H., Toroczkai, Z., Ercsey-Ravasz, M., Van Essen, D.C., Knoblauch, K., Burkhalter, A., 2018. The mouse cortical connectome, characterized by an ultra-dense cortical graph, maintains specificity by distinct connectivity profiles. Neuron 97, 698-715.e10. doi $10.1016 /$ J.NEURON.2017.12.037.

Guimerá, R., Amaral, L.A.N, 2005. Functional cartography of complex metabolic networks. Nature 433, 895-900. doi: $10.1038 /$ nature03288.

Harriger, L., van den Heuvel, M.P., Sporns, O., 2012. Rich club organization of macaque cerebral cortex and its role in network communication. PLoS One 7, e46497. doi:10.1371/journal . pone.0046497.

Harris, T.W., Antoshechkin, I., Bieri, T., Blasiar, D., Chan, J., et al., 2010. WormBase: a comprehensive resource for nematode research. Nucleic Acids Res. 38, D463-D467. doi:10.1093/nar/gkp952.

Hawrylycz, M., Miller, J.A., Menon, V., Feng, D., Dolbeare, T., et al.,2015. Canonical genetic signatures of the adult human brain. Nat. Neurosci. 18, 1832-1844. doi:10.1038/nn.4171.

Hawrylycz, M.J., Lein, E.S., Guillozet-Bongaarts, A.L., Shen, E.H., Ng, L., et al., 2012. An anatomically comprehensive atlas of the adult human brain transcriptome. Nature 489, 391-9. doi:10.1038/nature11405.

Henderson, J.a., Robinson, P.a., 2014. Relations between the geometry of cortical gyrification and white-matter network architecture. Brain Connect. 4, 112-30. doi:10.1089/brain.2013.0183.

van den Heuvel, M.P., Bullmore, E.T., Sporns, O., 2016a. Comparative Connectomics. Trends Cogn. Sci. $20,345-361$. doi: $10.1016 / j . t i c s .2016 .03 .001$.

van den Heuvel, M.P., Kahn, R.S., Goni, J., Sporns, O., 2012. High-cost, high-capacity backbone for global brain communication. Proc. Natl. Acad. Sci. 109, 11372-11377. doi:10.1073/pnas.1203593109

van den Heuvel, M.P., Scholtens, L.H., de Reus, M.A., 2016b. Topological organization of connectivity strength in the rat connectome. Brain Struct. Funct. 221, 1719-1736. doi:10.1007/s00429-015-0999-6.

van den Heuvel, M.P., van Soelen, I.L., Stam, C.J., Kahn, R.S., et Al., B., 2013. Genetic control of functional brain network efficiency in children. Eur. Neuropsychopharmacol. 23, 19-23. doi:10.1016/j.euroneuro. 2012. 06.007

van den Heuvel, M.P., Sporns, O., 2011. Rich-club organization of the human connectome. J. Neurosci. 31, 1577515786. doi:10 . 1523/ JNEUROSCI . 3539-11.2011.

van den Heuvel, M.P., Sporns, O., 2013. An anatomical substrate for integration among functional networks in human cortex. J. Neurosci. 33, 14489-14500. doi:10.1523/JNEUROSCI . 2128-13.2013.

Hervé, A., 2010. Partial least squares regression and projection on latent structure regression (PLS Regression). Wiley Interdiscip Rev Comput Stat 2,97-106. doi:10.1002/wics.51

Hu, G., Wang, G.Z., 2017. Decoding nervous system by single-cell RNA sequencing. Quant. Biol. 5, $210-214$. doi:10.1007/s40484-017-0116-3 
Hwang, K., Hallquist, M.N., Luna, B., 2013. The development of hub architecture in the human functional brain network. Cereb. Cortex 23, 2380-2393. doi:10.1093/cercor/bhs227

Irizarry, R.A., Wang, C., Zhou, Y., Speed, T.P., 2009. Gene set enrichment analysis made simple. Stat. Methods Med. Res. 18, 565-75. doi:10.1177/0962280209351908.

Jahanshad, N., Rajagopalan, P., Hua, X., Hibar, D.P., Nir, T.M., et al., 2013. Genome-wide scan of healthy human connectome discovers SPON1 gene variant influencing dementia severity. Proc. Natl. Acad. Sci. 110, 4768-73. doi:10.1073/pnas.1216206110.

Ji, S., Fakhry, A., Deng, H., 2014. Integrative analysis of the connectivity and gene expression atlases in the mouse brain. Neuroimage 84, 245-253. doi:10.1016/j.neuroimage.2013.08.049.

Johnson, G.A., Badea, A., Brandenburg, J., Cofer, G., Fubara, B., Liu, S., Nissanov, J., 2010. Waxholm Space: an image-based reference for coordinating mouse brain research. Neuroimage 53, 365-372. doi:10.1016/j. neuroimage.2010.06.067.

Johnson, M.B., Wang, P.P., Atabay, K.D., Murphy, E.A., Doan, R.N., Hecht, J.L., Walsh, C.A., 2015. Single-cell analysis reveals transcriptional heterogeneity of neural progenitors in human cortex. Nat. Neurosci. 18, 637-646. doi:10.1038/nn.3980.

Kanehisa, M., Goto, S., 2000. KEGG: kyoto encyclopedia of genes and genomes. Nucleic Acids Res. 28, $27-30$.

Kang, H.J., Kawasawa, Y.I., Cheng, F., Zhu, Y., Xu, X., et al.,2011. Spatio-temporal transcriptome of the human brain. Nature 478, 483-9. doi:10.1038/nature10523.

Kaufman, A., Dror, G., Meilijson, I., Ruppin, E., 2006. Gene expression of Caenorhabditis elegans neurons carries information on their synaptic connectivity. PLoS Comput. Biol. 2, e167. doi:10.1371/journal.pcbi. 0020167

Keil, J.M., Qalieh, A., Kwan, K.Y., 2018. Brain transcriptome databases: a user's guide. J. Neurosci. 10, $1930-17$. doi:10.1523/JNEUROSCI.1930-17.2018.

Keo, A., Aziz, N.A., Dzyubachyk, O., van der Grond, J., van Roon-Mom, W.M.C., Lelieveldt, B.P.F., Reinders, M.J.T., Mahfouz, A., 2017. Co-expression patterns between ATN1 and ATXN2 coincide with brain regions affected in Huntington's disease. Front. Mol. Neurosci. 10, 1-13. doi:10.3389/fnmol.2017.00399.

Klauser, P., Baker, S.T., Cropley, V.L., Bousman, C., Fornito, A., Cocchi, L., 2016. White matter disruptions in schizophrenia are spatially widespread and topologically converge on brain network hubs. Schizophr. Bull. sbw100, 1. doi:10.1093/schbul/sbw100.

Krienen, F.M., Yeo, B.T.T., Ge, T., Buckner, R.L., Sherwood, C.C., 2016. Transcriptional profiles of supragranularenriched genes associate with corticocortical network architecture in the human brain. Proc. Natl. Acad. Sci. 113, E469-78. doi:10.1073/pnas.1510903113.

Kumar, A., Gibbs, J.R., Beilina, A., Dillman, A., Kumaran, R., Trabzuni, D., Ryten, M., Walker, R., Smith, C., Traynor, B.J., Hardy, J., Singleton, A.B., Cookson, M.R., 2013. Age-associated changes in gene expression in human brain and isolated neurons. Neurobiol. Aging 34, 1199-209. doi:10.1016/j.neurobiolaging.2012.10.021.

Lein, E.S., Hawrylycz, M.J., Ao, N., Ayres, M., Bensinger, A., et al., 2007. Genome-wide atlas of gene expression in the adult mouse brain. Nature 445, 168-176. doi:10.1038/nature05453.

Liang, X., Zou, Q., He, Y., Yang, Y., 2013. Coupling of functional connectivity and regional cerebral blood flow reveals a physiological basis for network hubs of the human brain. Proc. Natl. Acad. Sci. 110, 1929-34. doi:10.1073/ pnas.1214900110

Lichtman, J.W., Denk, W., 2011. The big and the small: challenges of imaging the brain's circuits. Science. 334, 618-623. doi:10.1126/science.1209168. 
Liu, H., Bebu, I., Li, X., 2010. Microarray probes and probe sets. Front. Biosci. (Elite Ed). 2, 325-38. doi 10 . $1016 /$ j.biotechadv.2011.08.021.Secreted.

Mancarci, B.O., Toker, L., Tripathy, S.J., Li, B., Rocco, B., Sibille, E., Pavlidis, P., 2017. Cross-laboratory analysis of brain cell type transcriptomes with applications to interpretation of bulk tissue data. eNeuro 4, ENEURO.021217.2017. doi:10.1523/ENEURO.0212-17.2017.

Markett, S., de Reus, M.A., Reuter, M., Montag, C., Weber, B., Schoene-Bake, J.C., van den Heuvel, M.P., 2017. Variation on the dopamine D2 receptor gene (DRD2) is associated with basal ganglia-to-frontal structural connectivity. Neuroimage 155, 473-479. doi:10.1016/j.neuroimage.2017.04.005.

Markov, N.T., Ercsey-Ravasz, M.M., Ribeiro Gomes, A.R., Lamy, C., Magrou, L., Vezoli, J., Misery, P., Falchier, A., Quilodran, R., Gariel, M.A., Sallet, J., Gamanut, R., Huissoud, C., Clavagnier, S., Giroud, P., Sappey-Marinier, D., Barone, P., Dehay, C., Toroczkai, Z., Knoblauch, K., Van Essen, D.C., Kennedy, H., 2014. A weighted and directed interareal connectivity matrix for macaque cerebral cortex. Cereb. Cortex 24, 17-36. doi/10.1093/cercor/ bhs270.

McColgan, P., Gregory, S., Seunarine, K.K., Razi, A., Papoutsi, M., et al., 2018. Brain regions showing white matter loss in Huntington's disease are enriched for synaptic and metabolic genes. Biol. Psychiatry 83, 456-465. doi $10.1016 /$ J.BIOPSYCH.2017.10.019

Mesulam, M.M., 1998. From sensation to cognition. Brain a J. Neurol. 121, 1013-52.

Meunier, D., Lambiotte, R., Fornito, A., Ersche, K.D., Bullmore, E.T., 2009. Hierarchical modularity in human brain functional networks. Front. Neuroinform. 3,37. doi:10.3389/neuro.11.037.2009

Meyer, K., Damasio, A., 2009. Convergence and divergence in a neural architecture for recognition and memory. Trends Neurosci. 32, 376-382. doi $10.1016 /$ J.TINS.2009.04.002.

Miller, J.A., Ding, S.L., Sunkin, S.M., Smith, K.A., Ng, L., et al., 2014. Transcriptional landscape of the prenatal human brain. Nature 508, 199-206. doi:10.1038/nature13185.

Mišić, B., Betzel, R., Nematzadeh, A., Goñi, J., Griffa, A., Hagmann, P., Flammini, A., Ahn, Y.Y., Sporns, O., 2015. Cooperative and competitive spreading dynamics on the human connectome. Neuron 86, 1518-1529. doi $10.1016 /$ J.NEURON.2015.05.035.

Mortazavi, A., Williams, B.A., McCue, K., Schaeffer, L., Wold, B., 2008. Mapping and quantifying mammalian transcriptomes by RNA-Seq. Nat. Methods 5, 621-628. doi $10.1038 /$ nmeth.1226.

Myers, A.J., Gibbs, J.R., Webster, J.A., Rohrer, K., Zhao, A., Marlowe, L., Kaleem, M., Leung, D., Bryden, L., Nath, P., Zismann, V.L., Joshipura, K., Huentelman, M.J., Hu-Lince, D., Coon, K.D., Craig, D.W., Pearson, J.V., Holmans, P., Heward, C.B., Reiman, E.M., Stephan, D., Hardy, J., 2007. A survey of genetic human cortical gene expression. Nat. Genet. 39, 1494-1499. doi:10.1038/ng.2007.16.

Negi, S.K., Guda, C., 2017. Global gene expression profiling of healthy human brain and its application in studying neurological disorders. Sci. Rep. 7, 897. doi 10.1038/s41598-017-00952-9.

Oh, S.W., Harris, J.A., Ng, L., Winslow, B., Cain, N., et al., 2014. A mesoscale connectome of the mouse brain. Nature 508, 207-214. doi:10.1038/nature13186.

Okoniewski, M.J., Miller, C.J., 2006. Hybridization interactions between probesets in short oligo microarrays lead to spurious correlations. BMC Bioinformatics 7, 276. doi 10.1186/1471-2105-7-276.

Oldham, S., Fulcher, B., Parkes, L., Arnatkevičiūtè, A., Suo, C., Fornito, A., 2018. Consistency and differences between centrality metrics across distinct classes of networks URL: https://arxiv.org/pdf/1805.02375.pdf

Pantazatos, S.P., Li, X., 2017. Commentary: BRAIN NETWORKS. Correlated gene expression supports synchronous activity in brain networks. Science 348, 1241-4. Front. Neurosci. 11, 412. doi $10.3389 /$ fnins.2017.00412. 
Parkes, L., Fulcher, B.D., Yücel, M., Fornito, A., 2017. Transcriptional signatures of connectomic subregions of the human striatum. Genes, Brain Behav. 16, 647-663. doi $10.1111 /$ gbb.12386.

Paus, T., Keshavan, M., Giedd, J.N., 2008. Why do many psychiatric disorders emerge during adolescence? Nat. Rev. Neurosci. 9, 947-957.

Picardi, E., Horner, D.S., Pesole, G., 2017. Single-cell transcriptomics reveals specific RNA editing signatures in the human brain. RNA 23, 860-865. doi $10.1261 /$ rna.058271.116.

Poulin, J.F., Tasic, B., Hjerling-Leffler, J., Trimarchi, J.M., Awatramani, R., 2016. Disentangling neural cell diversity using single-cell transcriptomics. Nat. Neurosci. 19, 1131-1141. doi:10.1038/nn.4366

Power, J.D., Cohen, A.L., Nelson, S.M., Wig, G.S., Barnes, K.A., Church, J.A., Vogel, A.C., Laumann, T.O., Miezin, F.M., Schlaggar, B.L., Petersen, S.E., 2011. Functional network organization of the human brain. Neuron 72, 665-78. doi:10.1016/j.neuron.2011.09.006.

de Reus, M.A., van den Heuvel, M.P., 2013. Rich club organization and intermodule communication in the cat connectome. J. Neurosci. 33, 12929-12939. doi:10.1523/JNEUROSCI.1448-13.2013.

de Reus, M.A., van den Heuvel, M.P., 2014. Simulated rich club lesioning in brain networks: a scaffold for communication and integration? Front. Hum. Neurosci. 8, 647. doi:10.3389/fnhum.2014.00647.

Richiardi, J., Altmann, A., Milazzo, A.C., Chang, C., Chakravarty, M.M., et al., 2015. Correlated gene expression supports synchronous activity in brain networks. Science 348, 1241-1244. doi:10.1126/science. 1255905.

Rittman, T., Rubinov, M., Vértes, P.E., Patel, A.X., Ginestet, C.E., Ghosh, B.C., Barker, R.A., Spillantini, M.G., Bullmore, E.T., Rowe, J.B., 2016. Regional expression of the MAPT gene is associated with loss of hubs in brain networks and cognitive impairment in Parkinson disease and progressive supranuclear palsy. Neurobiol. Aging 48, 153-160. doi:10.1016/j.neurobiolaging.2016.09.001

Roberts, J.A., Perry, A., Lord, A.R., Roberts, G., Mitchell, P.B., Smith, R.E., Calamante, F., Breakspear, M., 2016. The contribution of geometry to the human connectome. Neuroimage 124,379-93. doi:10.1016/j.neuroimage. 2015.09 .009

Romero-Garcia, R., Whitaker, K.J., Váša, F., Seidlitz, J., Shinn, M., Fonagy, P., Dolan, R.J., Jones, P.B., Goodyer, I.M., Bullmore, E.T., Vértes, P.E., 2018. Structural covariance networks are coupled to expression of genes enriched in supragranular layers of the human cortex. Neuroimage 171, 256-267. doi:10.1016/J. NEUROIMAGE. 2017. 12.060

Romme, I.A.C., de Reus, M.A., Ophoff, R.A., Kahn, R.S., van den Heuvel, M.P., 2017. Connectome disconnectivity and cortical gene expression in patients with schizophrenia. Biol. Psychiatry 81, 495-502. doi:10.1016/j. biopsych.2016.07.012.

Royce, T.E., Rozowsky, J.S., Gerstein, M.B., 2007. Toward a universal microarray: prediction of gene expression through nearest-neighbor probe sequence identification. Nucleic Acids Res. 35, e99. doi $10.1093 / \mathrm{nar} / \mathrm{gkm} 549$

Rubinov, M., Ypma, R.J.F., Watson, C., Bullmore, E.T., 2015. Wiring cost and topological participation of the mouse brain connectome. Proc. Natl. Acad. Sci. 112, 10032-10037. doi:10.1073/pnas.1420315112.

Schröter, M., Paulsen, O., Bullmore, E.T., 2017. Micro-connectomics: probing the organization of neuronal networks at the cellular scale. Nat. Rev. Neurosci. 18, 131-146. doi $10.1038 / \mathrm{nrn} .2016 .182$

Schulze, A., Downward, J., 2001. Navigating gene expression using microarrays - a technology review. Nat. Cell Biol. 3, E190-E195. doi:10.1038/35087138.

Seguin, C., van den Heuvel, M.P., Zalesky, A., 2018. Navigation of brain networks. Proc. Natl. Acad. Sci. , 201801351 doi:10.1073/pnas.1801351115. 
Seidlitz, J., Váša, F., Shinn, M., Romero-Garcia, R., Whitaker, K.J., Vértes, P.E., Wagstyl, K., Kirkpatrick Reardon, P., Clasen, L., Liu, S., Messinger, A., Leopold, D.A., Fonagy, P., Dolan, R.J., Jones, P.B., Goodyer, I.M., Raznahan, A., Bullmore, E.T., 2018. Morphometric similarity networks detect microscale cortical organization and predict inter-individual cognitive variation. Neuron 97, 231-247.e7. doi:10.1016/j.neuron.2017.11.039

Senden, M., Deco, G., de Reus, M.A., Goebel, R., van den Heuvel, M.P., 2014. Rich club organization supports a diverse set of functional network configurations. Neuroimage 96, 174-182. doi:10.1016/j.neuroimage. 2014.03 .066

Sinclair, B., Hansell, N.K., Blokland, G.A.M., Martin, N.G., Thompson, P.M., Breakspear, M., de Zubicaray, G.I., Wright, M.J., McMahon, K.L., 2015. Heritability of the network architecture of intrinsic brain functional connectivity. Neuroimage 121, 243-52. doi $10.1016 / j$.neuroimage.2015.07.048.

Smit, D.J.A., Stam, C.J., Posthuma, D., Boomsma, D.I., de Geus, E.J.C., 2008. Heritability of "small-world" networks in the brain: a graph theoretical analysis of resting-state EEG functional connectivity. Hum. Brain Mapp. 29, 1368-1378. doi $10.1002 / \mathrm{hbm} .20468$.

Sporns, O., 2011. The non-random brain: efficiency, economy, and complex dynamics. Front. Comput. Neurosci. 5, 5. doi: $10.3389 /$ fncom.2011.00005.

Sporns, O., Honey, C.J., Kötter, R., 2007. Identification and classification of hubs in brain networks. PLoS One 2, e1049. doi:10.1371/ journal.pone.0001049.

Stephan, K.E., Kamper, L., Bozkurt, A., Burns, G.A., Young, M.P., Kötter, R., 2001. Advanced database methodology for the Collation of Connectivity data on the Macaque brain (CoCoMac). Philos. Trans. R. Soc. Lond. B. Biol. Sci. 356, 1159-86. doi $10.1098 /$ rstb.2001.0908.

Subramanian, A., Tamayo, P., Mootha, V.K., Mukherjee, S., Ebert, B.L., Gillette, M.A., Paulovich, A., Pomeroy, S.L., Golub, T.R., Lander, E.S., Mesirov, J.P., 2005. Gene set enrichment analysis: a knowledge-based approach for interpreting genome-wide expression profiles. Proc. Natl. Acad. Sci. 102, 15545-50. doi:10.1073/pnas. 0506580102

Tang, F., Barbacioru, C., Wang, Y., Nordman, E., Lee, C., Xu, N., Wang, X., Bodeau, J., Tuch, B.B., Siddiqui, A., Lao, K., Surani, M.A., 2009. mRNA-Seq whole-transcriptome analysis of a single cell. Nat. Methods 6, 377-382. doi:10.1038/nmeth.1315

Tasic, B., Menon, V., Nguyen, T.N., Kim, T.K., Jarsky, T., Yao, Z., Levi, B., Gray, L.T., Sorensen, S.A., Dolbeare, T., Bertagnolli, D., Goldy, J., Shapovalova, N., Parry, S., Lee, C., Smith, K., Bernard, A., Madisen, L., Sunkin, S.M., Hawrylycz, M., Koch, C., Zeng, H., 2016. Adult mouse cortical cell taxonomy revealed by single cell transcriptomics. Nat. Neurosci. 19, 335-346. doi $10.1038 / \mathrm{nn} .4216$.

Tomasi, D., Wang, G.J., Volkow, N.D., 2013. Energetic cost of brain functional connectivity. Proc. Natl. Acad. Sci. 110,13642-13647. doi:10.1073/pnas.1303346110.

Towlson, E.K., Vértes, P.E., Ahnert, S.E., Schafer, W.R., Bullmore, E.T., 2013. The rich club of the C. elegans neuronal connectome. J. Neurosci. 33, 6380-7. doi:10.1523/JNEUROSCI . 3784-12.2013.

Trabzuni, D., Ramasamy, A., Imran, S., Walker, R., Smith, C., Weale, M.E., Hardy, J., Ryten, M., Consortium, N.A.B.E., 2013. Widespread sex differences in gene expression and splicing in the adult human brain. Nat. Commun. 4, 2771. doi $10.1038 /$ ncomms 3771 .

Unger, E.R., Nitta, H., Lee, D.R., Grogan, T.M., 2010. In Situ Hybridization: principles and applications, in: Mol. Diagnostics. Elsevier, pp. 71-79. doi:10.1016/B978-0-12-369428-7.00007-0.

Vaishnavi, S.N., Vlassenko, A.G., Rundle, M.M., Snyder, A.Z., Mintun, M.A., Raichle, M.E., 2010. Regional aerobic glycolysis in the human brain. Proc. Natl. Acad. Sci. 107, 17757-62. doi $10.1073 /$ pnas.1010459107.

Van Den Heuvel, M.P., Sporns, O., Collin, G., Scheewe, T., Mandl, R.C., Cahn, W., Goni, J., Pol, H.E., Kahn, R.S., 2013. Abnormal rich club organization and functional brain dynamics in schizophrenia. JAMA Psychiatry 70 , 783-792. doi:10.1001/jamapsychiatry.2013.1328 
Varadan, V., Miller, D.M., Anastassiou, D., 2006. Computational inference of the molecular logic for synaptic connectivity in C. elegans. Bioinformatics 22, e497-506. doi:10.1093/bioinformatics/bt 1224

Varshney, L.R., Chen, B.L., Paniagua, E., Hall, D.H., Chklovskii, D.B., 2011. Structural properties of the Caenorhabditis elegans neuronal network. PLoS Comput. Biol. 7, e1001066. doi:10.1371/journal.pcbi.1001066.

Vértes, P.E., Rittman, T., Whitaker, K.J., Romero-Garcia, R., Váša, F., Kitzbichler, M.G., Wagstyl, K., Fonagy, P., Dolan, R.J., Jones, P.B., Goodyer, I.M., Bullmore, E.T., 2016. Gene transcription profiles associated with intermodular hubs and connection distance in human functional magnetic resonance imaging networks. Philos. Trans. R. Soc. B Biol. Sci. 371, 735-769. doi $10.1098 /$ rstb.2015.0362.

Wang, Z., Gerstein, M., Snyder, M., 2009. RNA-Seq: a revolutionary tool for transcriptomics. Nat. Rev. Genet. 10, 57-63. doi: 10.1038 /nrg2484

Watts, D.J., Strogatz, S.H., 1998. Collective dynamics of "small-world" networks. Nature 393, 440-442. doi: 10 . $1038 / 30918$.

Whitaker, K.J., Vértes, P.E., Romero-Garcia, R., Váša, F., Moutoussis, M., et al., 2016. Adolescence is associated with genomically patterned consolidation of the hubs of the human brain connectome. Proc. Natl. Acad. Sci. 113, 9105-10. doi:10.1073/pnas.1601745113.

White, J.G., Southgate, E., Thomson, J.N., Brenner, S., 1986. The structure of the nervous system of the nematode Caenorhabditis elegans. Philos. Trans. R. Soc. B 314, 1-340. doi:10.1098/rstb.1986.0056.

Wolf, L., Goldberg, C., Manor, N., Sharan, R., Ruppin, E., 2011. Gene expression in the rodent brain is associated with its regional connectivity. PLoS Comput. Biol. 7, e1002040. doi:10.1371/ journal.pcbi.1002040.

Yu, P., Lin, W., 2016. Single-cell transcriptome study as big data. Genomics. Proteomics Bioinformatics 14, 21-30. doi:10.1016/j.gpb.2016.01.005.

Zalesky, A., Fornito, A., Bullmore, E., 2012. On the use of correlation as a measure of network connectivity. Neuroimage 60, 2096-2106. doi:10.1016/J.NEUROIMAGE.2012.02.001.

Zamora-López, G., Zhou, C., Kurths, J., 2010. Cortical hubs form a module for multisensory integration on top of the hierarchy of cortical networks. Front. Neuroinform. 4, 1. doi:10.3389/neuro.11.001.2010

Zhan, L., Jahanshad, N., Faskowitz, J., Zhu, D., Prasad, G., Martin, N.G., de Zubicaray, G.I., McMahon, K.L., Wright, M.J., Thompson, P.M., 2015. Heritability of brain network topology in 853 twins and siblings. Proc. / IEEE Int. Symp. Biomed. Imaging from nano to macro , 449 doi:10.1109/ISBI.2015.7163908.

Zhong, S., Zhang, S., Fan, X., Wu, Q., Yan, L., Dong, J., Zhang, H., Li, L., Sun, L., Pan, N., Xu, X., Tang, F., Zhang, J., Qiao, J., Wang, X., 2018. A single-cell RNA-seq survey of the developmental landscape of the human prefrontal cortex. Nature 555, 524-528. doi: 10.1038 / nature25980 